FORMALIZED MATHEMATICS

Vol. 21, No. 4, Pages 241-247, 2013

DOI: 10.2478 /forma-2013-0026
DE GRUYTER

OPEN

degruyter.com/view/j/forma

\title{
Formulation of Cell Petri Nets
}

\author{
Mitsuru Jitsukawa \\ Shinshu University \\ Nagano, Japan
}

\author{
Pauline N. Kawamoto \\ Shinshu University \\ Nagano, Japan
}

\author{
Yasunari Shidama \\ Shinshu University \\ Nagano, Japan
}

Summary. Based on the Petri net definitions and theorems already formalized in the Mizar article [13, in this article we were able to formalize the definition of cell Petri nets. It is based on [12]. Colored Petri net has already been defined in 11. In addition, the conditions of the firing rule and the colored set to this definition, that defines the cell Petri nets are further extended to CPNT.i further. The synthesis of two Petri nets was introduced in [1] and in this work the definition is extended to produce the synthesis of a family of colored Petri nets. Specifically, the extension to a CPNT family is performed by specifying how to link the outbound transitions of each colored Petri net to the place elements of other nets to form a neighborhood relationship. Finally, the activation of colored Petri nets was formalized.

MSC: 68-04 03B35

Keywords: Petri net; system modelling

MML identifier: PETRI_3, version: 8.1.02 5.22.1191

The notation and terminology used in this paper have been introduced in the following articles: [1], [15], [10], [5], 6], 7], [17], [2], 3], [4], 8], [16], [13], [1], [19], [14], [18], and [9].

\section{Preliminaries}

Let $I$ be a non empty set and $C_{1}$ be a many sorted set indexed by $I$. We say that $C_{1}$ is colored Petri net family-like if and only if

(Def. 1) Let us consider an element $i$ of $I$. Then $C_{1}(i)$ is a colored place/transition net.

Note that there exists a many sorted set indexed by $I$ which is colored Petri net family-like.

A colored Petri net family of $I$ is a colored Petri net family-like many sorted set indexed by $I$. Let $C_{1}$ be a colored Petri net family of $I$ and $i$ be an element 
of $I$. One can check that the functor $C_{1}(i)$ yields a colored place/transition net. Let $C_{2}$ be a colored Petri net family of $I$. We say that $C_{2}$ is disjoint valued if and only if

(Def. 2) Let us consider elements $i, j$ of $I$. Suppose $i \neq j$. Then

(i) the carrier of $C_{2}(i)$ misses the carrier of $C_{2}(j)$, and

(ii) the carrier' of $C_{2}(i)$ misses the carrier' of $C_{2}(j)$.

Now we state the propositions:

(1) Let us consider a set $I$ and many sorted sets $F, D, R$ indexed by $I$. Suppose

(i) for every element $i$ such that $i \in I$ there exists a function $f$ such that $f=F(i)$ and $\operatorname{dom} f=D(i)$ and $\operatorname{rng} f=R(i)$, and

(ii) for every elements $i, j$ and for every functions $f, g$ such that $i, j \in I$ and $i \neq j$ and $f=F(i)$ and $g=F(j)$ holds $\operatorname{dom} f$ misses $\operatorname{dom} g$.

Then there exists a function $G$ such that

(iii) $G=\bigcup \operatorname{rng} F$, and

(iv) $\operatorname{dom} G=\bigcup \operatorname{rng} D$, and

(v) $\operatorname{rng} G=\bigcup \operatorname{rng} R$, and

(vi) for every elements $i, x$ and for every function $f$ such that $i \in I$ and $f=F(i)$ and $x \in \operatorname{dom} f$ holds $G(x)=f(x)$.

Proof: For every element $z$ such that $z \in \bigcup \operatorname{rng} F$ there exist elements $x, y, i$ such that $z=\langle x, y\rangle$ and $z \in F(i)$ and $i \in I$. For every element $z$ such that $z \in \bigcup \operatorname{rng} F$ there exist elements $x, y$ such that $z=\langle x, y\rangle$. Reconsider $G=\bigcup \operatorname{rng} F$ as a binary relation. $G$ is a function. For every element $x, x \in \operatorname{dom} G$ iff $x \in \bigcup \operatorname{rng} D$ by [5, (3)]. For every element $x$, $x \in \operatorname{rng} G$ iff $x \in \bigcup \operatorname{rng} R$ by [5, (3)]. For every elements $i, x$ and for every function $f$ such that $i \in I$ and $f=F(i)$ and $x \in \operatorname{dom} f$ holds $G(x)=f(x)$ by [5, (1), (3)].

(2) Let us consider a set $I$ and many sorted sets $Y, Z$ indexed by $I$. Suppose elements $i, j$. If $i, j \in I$ and $i \neq j$, then $Y(i) \cap Z(j)=\emptyset$. Then $\bigcup(Y \backslash Z)=$ $\cup Y \backslash \cup Z$. Proof: Set $X=Y \backslash Z$. For every element $x, x \in \bigcup \operatorname{rng} X$ iff $x \in \bigcup \operatorname{rng} Y \backslash \bigcup \operatorname{rng} Z$ by [5, (3)].

(3) Let us consider a set $I$ and many sorted sets $X, Y, Z$ indexed by $I$. Suppose

(i) $X \subseteq Y \backslash Z$, and

(ii) for every elements $i, j$ such that $i, j \in I$ and $i \neq j$ holds $Y(i) \cap$ $Z(j)=\emptyset$.

Then $\bigcup X \subseteq \cup Y \backslash \cup Z$. The theorem is a consequence of (2). 


\section{SyNTHESIS OF CPNT AND I}

Let $I$ be a non trivial set. The functor XorDelta $I$ yielding a non empty set is defined by the term

(Def. 3) $\{\langle i, j\rangle$, where $i, j$ are elements of $I: i \neq j\}$.

Now we state the proposition:

(4) Let us consider a non trivial finite set $I$ and a colored Petri net family $C_{2}$ of $I$. Then $\bigcup\left\{\left(\text { the carrier of } C_{2}(j)\right)^{\text {Outbds }\left(C_{2}(i)\right)}\right.$, where $i, j$ are elements of $I: i \neq j\}$ is not empty.

Let $I$ be a non trivial finite set and $C_{2}$ be a colored Petri net family of $I$. A connecting mapping of $C_{2}$ is a many sorted set indexed by XorDelta $I$ and is defined by

(Def. 4) (i) rng it $\subseteq \bigcup\left\{\left(\text { the carrier of } C_{2}(j)\right)^{\text {Outbds }\left(C_{2}(i)\right)}\right.$, where $i, j$ are elements of $I: i \neq j\}$, and

(ii) for every elements $i, j$ of $I$ such that $i \neq j$ holds $i t(\langle i, j\rangle)$ is a function from Outbds $\left(C_{2}(i)\right)$ into the carrier of $C_{2}(j)$.

Now we state the proposition:

(5) Let us consider colored place/transition nets $C_{4}, C_{5}$, a function $O_{1}$ from Outbds $C_{4}$ into the carrier of $C_{5}$, and a function $q_{1}$. Suppose

(i) $\operatorname{dom} q_{1}=$ Outbds $C_{4}$, and

(ii) for every transition $t_{1}$ of $C_{4}$ such that $t_{1}$ is outbound holds $q_{1}\left(t_{1}\right)$ is a function from the thin cylinders of the colored set of $C_{4}$ and ${ }^{*}\left\{t_{1}\right\}$ into the thin cylinders of the colored set of $C_{4}$ and $O_{1}{ }^{\circ} t_{1}$.

Then $q_{1} \in\left(\bigcup\left\{\text { the thin cylinders of the colored set of } C_{4} \text { and } O_{1}{ }^{\circ} t_{1}\right)^{\alpha}\right.$, where $t_{1}$ is a transition of $C_{4}: t_{1}$ is outbound $\left.\}\right)^{\text {Outbds } C_{4}}$, where $\alpha$ is the thin cylinders of the colored set of $C_{4}$ and ${ }^{*}\left\{t_{1}\right\}$.

Let $I$ be a non trivial finite set, $C_{2}$ be a colored Petri net family of $I$, and $O$ be a connecting mapping of $C_{2}$. A connecting firing rule of $O$ is a many sorted set indexed by XorDelta $I$ and is defined by

(Def. 5) Let us consider elements $i, j$ of $I$. Suppose $i \neq j$. Then there exists a function $\mathrm{O}_{2}$ from $\operatorname{Outbds}\left(C_{2}(i)\right)$ into the carrier of $C_{2}(j)$ and there exists a function $q_{2}$ such that $q_{2}=i t(\langle i, j\rangle)$ and $O_{2}=O(\langle i, j\rangle)$ and $\operatorname{dom} q_{2}=\operatorname{Outbds}\left(C_{2}(i)\right)$ and for every transition $t_{1}$ of $C_{2}(i)$ such that $t_{1}$ is outbound holds $q_{2}\left(t_{1}\right)$ is a function from the thin cylinders of the colored set of $C_{2}(i)$ and $*\left\{t_{1}\right\}$ into the thin cylinders of the colored set of $C_{2}(i)$ and $O_{2}{ }^{\circ} t_{1}$. 


\section{Extension to a Family of Colored Petri Nets}

Let $I$ be a non trivial finite set, $C_{2}$ be a colored Petri net family of $I, O$ be a connecting mapping of $C_{2}$, and $q$ be a connecting firing rule of $O$. Assume $C_{2}$ is disjoint valued and for every elements $i, j_{1}, j_{2}$ of $I$ such that $i \neq j_{1}$ and $i \neq j_{2}$ and there exist elements $x, y_{1}, y_{2}$ such that $\left\langle x, y_{1}\right\rangle \in q\left(\left\langle i, j_{1}\right\rangle\right)$ and $\langle x$, $\left.y_{2}\right\rangle \in q\left(\left\langle i, j_{2}\right\rangle\right)$ holds $j_{1}=j_{2}$. The functor synthesis $q$ yielding a strict colored place/transition net is defined by

(Def. 6) There exist many sorted sets $P, T, S_{1}, T_{1}, C_{3}, F$ indexed by $I$ and there exist functions $U, U_{1}$ such that for every element $i$ of $I, P(i)=$ the carrier of $C_{2}(i)$ and $T(i)=$ the carrier' of $C_{2}(i)$ and $S_{1}(i)=$ the S-T $\operatorname{arcs}$ of $C_{2}(i)$ and $T_{1}(i)=$ the T-S arcs of $C_{2}(i)$ and $C_{3}(i)=$ the colored set of $C_{2}(i)$ and $F(i)=$ the firing rule of $C_{2}(i)$ and $U=\bigcup \operatorname{rng} F$ and $U_{1}=\bigcup \operatorname{rng} q$ and the carrier of $i t=\bigcup \operatorname{rng} P$ and the carrier' of it $=\bigcup \operatorname{rng} T$ and the S-T arcs of $i t=\bigcup \operatorname{rng} S_{1}$ and the T-S arcs of it $=\bigcup \operatorname{rng} T_{1} \cup \bigcup \operatorname{rng} O$ and the colored set of $i t=\bigcup \operatorname{rng} C_{3}$ and the firing rule of $i t=U+\cdot U_{1}$.

\section{Definition of Cell Petri Nets}

Let $I$ be a non empty finite set and $C_{2}$ be a colored Petri net family of $I$. We say that $C_{2}$ is cell Petri nets if and only if

(Def. 7) There exists a function $N$ from $I$ into $2^{\text {rng } C_{2}}$ such that for every element $i$ of $I, N(i)=\left\{C_{2}(j)\right.$, where $j$ is an element of $\left.I: j \neq i\right\}$.

Let $N$ be a function from $I$ into $2^{\text {rng } C_{2}}$ and $O$ be a connecting mapping of $C_{2}$. We say that $(N, O)$ is cell Petri nets if and only if

(Def. 8) Let us consider an element $i$ of $I$. Then $N(i)=\left\{C_{2}(j)\right.$, where $j$ is an element of $I: j \neq i$ and there exists a transition $t$ of $C_{2}(i)$ and there exists an element $s$ such that $\langle t, s\rangle \in O(\langle i, j\rangle)\}$.

Now we state the proposition:

(6) Let us consider a non trivial finite set $I$, a colored Petri net family $C_{2}$ of $I$, a function $N$ from $I$ into $2^{\text {rng } C_{2}}$, and a connecting mapping $O$ of $C_{2}$. Suppose

(i) $C_{2}$ is one-to-one, and

(ii) $(N, O)$ is cell Petri nets.

Let us consider an element $i$ of $I$. Then $C_{2}(i) \notin N(i)$. 


\section{Activation of Petri Nets}

Let $C_{6}$ be a colored place/transition net structure. We say that $C_{6}$ has nontrivial colored set if and only if

(Def. 9) The colored set of $C_{6}$ is not trivial.

One can verify that there exists a strict colored-PT-net-like colored Petri net which has nontrivial colored set.

Let $C_{2}$ be a colored place/transition net with nontrivial colored set. One can verify that the colored set of $C_{2}$ is non trivial.

Let $C_{6}$ be a colored place/transition net with nontrivial colored set, $S$ be a subset of the carrier of $C_{6}$, and $D$ be a thin cylinder of the colored set of $C_{6}$ and $S$. A color threshold of $D$ is a function from loc $D$ into the colored set of $C_{6}$. Let $C_{6}$ be a colored place/transition net. A color count of $C_{6}$ is a function from the colored set of $C_{6}$ into $\mathbb{N}$. The colored states of $C_{6}$ yielding a non empty set is defined by the term

(Def. 10) the set of all $e$ where $e$ is a color count of $C_{6}$.

A colored state of $C_{6}$ is a function from $C_{6}$ into the colored states of $C_{6}$. From now on $C_{6}$ denotes a colored place/transition net with nontrivial colored set, $m$ denotes a colored state of $C_{6}$, and $t$ denotes an element of the carrier' of $C_{6}$.

Let $C_{6}$ be a colored place/transition net with nontrivial colored set, $m$ be a colored state of $C_{6}$, and $p$ be a place of $C_{6}$. Observe that the functor $m(p)$ yields a color count of $C_{6}$. Let $m_{1}$ be a color count of $C_{6}$ and $x$ be an element. Let us observe that the functor $m_{1}(x)$ yields an element of $\mathbb{N}$. Let us consider $C_{6}, m$, and $t$. Let $D$ be a thin cylinder of the colored set of $C_{6}$ and ${ }^{*}\{t\}$ and $C_{7}$ be a color threshold of $D$. We say that $t$ is firable on $m$ and $C_{7}$ if and only if

(Def. 11) (i) (the firing rule of $\left.C_{6}\right)(\langle t, D\rangle) \neq \emptyset$, and

(ii) for every place $p$ of $C_{6}$ such that $p \in \operatorname{loc} D$ holds $1 \leqslant m(p)\left(C_{7}(p)\right)$.

The firable set on $m$ and $t$ yielding a set is defined by the term

(Def. 12) $\left\{D\right.$, where $D$ is a thin cylinder of the colored set of $C_{6}$ and ${ }^{*}\{t\}$ : there exists a color threshold $C_{7}$ of $D$ such that $t$ is firable on $m$ and $\left.C_{7}\right\}$.

Now we state the proposition:

(7) Let us consider a thin cylinder $D$ of the colored set of $C_{6}$ and ${ }^{*}\{t\}$. Then there exists a color threshold $C_{7}$ of $D$ such that $t$ is firable on $m$ and $C_{7}$ if and only if $D \in$ the firable set on $m$ and $t$.

Let us consider $C_{6}, m$, and $t$. Let $D$ be a thin cylinder of the colored set of $C_{6}$ and ${ }^{*}\{t\}, C_{7}$ be a color threshold of $D$, and $p$ be an element of $C_{6}$. Assume $t$ is firable on $m$ and $C_{7}$. The Petri subtraction $\left(C_{7}, m, p\right)$ yielding a function from the colored set of $C_{6}$ into $\mathbb{N}$ is defined by

(Def. 13) Let us consider an element $x$ of the colored set of $C_{6}$. Then 
(i) if $p \in \operatorname{loc} D$ and $x=C_{7}(p)$, then it $(x)=m(p)(x)-1$, and

(ii) if it is not true that $p \in \operatorname{loc} D$ and $x=C_{7}(p)$, then $i t(x)=m(p)(x)$.

Let $D$ be a thin cylinder of the colored set of $C_{6}$ and $\overline{\{t\}}$. The Petri addition $\left(C_{7}, m, p\right)$ yielding a function from the colored set of $C_{6}$ into $\mathbb{N}$ is defined by

(Def. 14) Let us consider an element $x$ of the colored set of $C_{6}$. Then

(i) if $p \in \operatorname{loc} D$ and $x=C_{7}(p)$, then it $(x)=m(p)(x)+1$, and

(ii) if it is not true that $p \in \operatorname{loc} D$ and $x=C_{7}(p)$, then it $(x)=m(p)(x)$.

Let $D$ be a thin cylinder of the colored set of $C_{6}$ and ${ }^{*}\{t\}$ and $E$ be a thin cylinder of the colored set of $C_{6}$ and $\overline{\{t\}}$. Let $C_{10}$ be a color threshold of $E$. The firing result $\left(C_{7}, C_{10}, m, p\right)$ yielding a function from the colored set of $C_{6}$ into $\mathbb{N}$ is defined by the term

(Def. 15)

$$
\left\{\begin{aligned}
\text { the Petri subtraction }\left(C_{7}, m, p\right), & \\
& \text { if } t \text { is firable on } m \text { and } C_{7}, \text { and } p \in \operatorname{loc} D \backslash \operatorname{loc} E, \\
\text { the Petri addition }\left(C_{10}, m, p\right), & \\
& \text { if } t \text { is firable on } m \text { and } C_{7}, \text { and } p \in \operatorname{loc} E \backslash \operatorname{loc} D, \\
m(p), & \text { otherwise. }
\end{aligned}\right.
$$

Let us consider a thin cylinder $D_{1}$ of the colored set of $C_{6}$ and $*\{t\}$, a thin cylinder $D_{2}$ of the colored set of $C_{6}$ and $\overline{\{t\}}$, a color threshold $C_{8}$ of $D_{1}$, a color threshold $C_{9}$ of $D_{2}$, an element $x$ of the colored set of $C_{6}$, and an element $p$ of $C_{6}$. Now we state the propositions:

(8) $m(p)(x)-1 \leqslant\left(\right.$ the firing result $\left.\left(C_{8}, C_{9}, m, p\right)\right)(x) \leqslant m(p)(x)+1$.

(9) If $t$ is outbound, then $m(p)(x)-1 \leqslant\left(\right.$ the firing result $\left.\left(C_{8}, C_{9}, m, p\right)\right)(x) \leqslant$ $m(p)(x)$.

Acknowledgement: We are thankful to Dr. Yatsuka Nakamura. He is the former professor of the Shinshu University. The completion of this article would not have be possible without the deep insight into the automatic proof verification system of Dr. Nakamura. Thank you.

\section{REFERENCES}

[1] Grzegorz Bancerek. König's theorem. Formalized Mathematics, 1(3):589-593, 1990.

[2] Grzegorz Bancerek. Free term algebras. Formalized Mathematics, 20(3):239-256, 2012. doi $10.2478 /$ v10037-012-0029-6

[3] Grzegorz Bancerek. The fundamental properties of natural numbers Formalized Mathematics, 1(1):41-46, 1990.

[4] Grzegorz Bancerek. The ordinal numbers. Formalized Mathematics, 1(1):91-96, 1990.

[5] Czesław Byliński. Functions and their basic properties. Formalized Mathematics, 1(1): 55-65, 1990.

[6] Czesław Byliński. Functions from a set to a set. Formalized Mathematics, 1(1):153-164, 1990.

[7] Czesław Byliński. The modification of a function by a function and the iteration of the composition of a function. Formalızed Mathematıcs, 1(3):521-527, 1990. 
[8] Czesław Byliński. Partial functions. Formalized Mathematics, 1(2):357-367, 1990.

[9] Czesław Byliński. Some basic properties of sets. Formalized Mathematics, 1(1):47-53, 1990.

[10] Agata Darmochwał. Finite sets Formalized Mathematics, 1(1):165-167, 1990.

[11] Mitsuru Jitsukawa, Pauline N. Kawamoto, Yasunari Shidama, and Yatsuka Nakamura. Cell Petri net concepts. Formalized Mathematics, 17(1):37-42, 2009. doi:10.2478/v10037009-0004-z

[12] Pauline N. Kawamoto and Yatsuka Nakamura. On Cell Petri Nets. Journal of Applied Functional Analysis, 1996.

[13] Pauline N. Kawamoto, Yasushi Fuwa, and Yatsuka Nakamura. Basic Petri net concepts Formalized Mathematics, 3(2):183-187, 1992.

[14] Krzysztof Retel. Properties of first and second order cutting of binary relations Formalized Mathematics, 13(3):361-365, 2005.

[15] Andrzej Trybulec. Domains and their Cartesian products Formalized Mathematics, 1(1): 115-122, 1990.

[16] Andrzej Trybulec. Many sorted sets. Formalized Mathematics, 4(1):15-22, 1993.

[17] Michał J. Trybulec. Integers. Formalized Mathematics, 1(3):501-505, 1990.

[18] Zinaida Trybulec. Properties of subsets. Formalized Mathematics, 1(1):67-71, 1990.

[19] Edmund Woronowicz. Relations and their basic properties Formalized Mathematics, 1 (1):73-83, 1990.

Received December 8, 2013 